Article

\title{
Performance Analysis of Cooperative Non-Orthogonal Multiple Access in Visible Light Communication
}

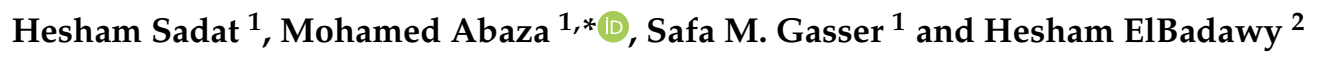 \\ 1 Arab Academy for Science, Technology and Maritime Transport, Cairo 2033, Egypt; \\ h.sadat20@student.aast.edu (H.S.); safagasser@aast.edu (S.M.G.) \\ 2 National Telecommunication Institute, NTI, Cairo 11768, Egypt; heshamelbadwy@ieee.org \\ * Correspondence: mohamed.abaza@aast.edu; Tel.: +20-122-444-2136
}

Received: 11 August 2019; Accepted: 16 September 2019; Published: 25 September 2019

check for updates

\begin{abstract}
Nowadays, visible light communication (VLC) systems have become one of the candidate technologies for high data rate indoor communications. However, the main challenge to develop a high data rate VLC system is the narrow modulation bandwidth of light-emitting diodes (LEDs). Power domain non-orthogonal multiple access (PD-NOMA) is a promising scheme to enhance the spectral efficiency of downlink VLC systems. In this paper, we introduce cooperative PD-NOMA to the system to improve the signal reception for the far users. We evaluate the bit error rate (BER) and achievable rate performance of non-cooperative and cooperative PD-NOMA under perfect channel state information (CSI). Moreover, we drive analytic expressions for the BER and provide a Monte Carlo simulation results for verifying the validity of the derived analytical BER results. The results show that cooperative PD-NOMA outperforms non-cooperative PD-NOMA by $8.2 \mathrm{~dB}$ at BER $10^{-6}$ and by achievable rate $14.1 \mathrm{bit} / \mathrm{s} / \mathrm{Hz}$ at $45 \mathrm{~dB}$ in a two-user scenario.
\end{abstract}

Keywords: visible light communication (VLC); power domain non-orthogonal multiple access (PD-NOMA); cooperative PD-NOMA; bit error rate (BER); achievable rate

\section{Introduction}

Visible light communication (VLC) is an attractive complementary wireless communication technique to conventional radio frequency (RF), especially in indoor environments due to its high data rate capability. VLC exploits light-emitting diodes (LEDs) lighting infrastructure which provides illumination and data transmission simultaneously [1]. VLC is an economical and secure system that can provide a high quality of service (QoS) with no electromagnetic interference, particularly in indoor environments [2,3].

One of the main advantages of LEDs is their ability to transmit data by varying the light intensity at very high frequency without being perceived by the human eye. This process is called intensity modulation (IM). At the receiver end, in a process known as direct detection (DD), a photodetector (PD) is used to generate an electrical current proportional to the variation in the received optical power [4]. The most commonly used IM technique is the on-off keying (OOK) modulation scheme due its implementation simplicity [5].

In spite of the above remarkable advantages, the major limitation to developing a high data rate link in VLC is the narrow modulation bandwidth of LEDs (several megahertz), which is a hardware issue [2]. Nevertheless, to overcome this constraint, multiple access techniques are used [6]. In [7], the authors discuss several multiple-access techniques to improve the downlink VLC data rate, including orthogonal multiple-access (OMA) techniques such as time division multiple access (TDMA), code division multiple access (CDMA) and orthogonal frequency division multiple access (OFDMA). 
On the other hand, power domain non-orthogonal multiple access (PD-NOMA) has an advantage over other conventional OMA techniques because different users can share the same time and frequency resources in the power domain, and thereby increase the spectral efficiency $[8,9]$. At the transmitter end, superposition coding is used to superimpose the signals of different users in the power domain by allocating a certain power level to each user based on its corresponding channel condition.

PD-NOMA allocates low power levels to users with good channel conditions, and high power levels to users with bad channel conditions. Therefore, at the receiver end, the user with bad channel conditions (highest power allocation) can directly decode its signal and treats other signals as noise. Meanwhile, other users have to apply successive interference cancellation (SIC) for multiuser signals separation before decoding their own signals [8].

Several researchers report on the PD-NOMA-VLC performance in comparison to other OMA techniques. The authors in $[10,11]$ investigate the ergodic sum rate of PD-NOMA-VLC. Their results show that PD-NOMA outperforms TDMA in terms of system capacity. The authors in [12] illustrate the superior performance of PD-NOMA-VLC over OFDMA scheme in terms of achievable data rate. Thus, one can conclude that PD-NOMA is an attractive multiple-access technique in VLC systems.

The authors in $[13,14]$ propose cooperative relaying as a promising candidate that improves the link reliability, and that extends the communication systems range. The authors in [15] apply PD-NOMA to multiple-antenna relaying networks to study the outage behavior of mobile users. Their results show that PD-NOMA improves spectral efficiency and fairness of the system compared to OMA schemes. Consequently, the authors in [16] propose a cooperative PD-NOMA for 5G systems where the user with good channel condition acts as a relay that retransmits data of the users with bad channel conditions by exploiting the prior information acquired from the SIC process.

In addition to the downlink transmission phase in PD-NOMA, the user with good channel condition acts as decode and forward (DF) relay for other users with bad channel conditions to enhance the signal reception reliability. Users cooperate and transmit data by using short-range communication technologies, like ultra-wideband and Bluetooth. Otherwise, users with good channel conditions require extra time slots to deliver data to those with bad channel conditions [16].

To the best of our knowledge, this paper is the first to investigate the bit error rate (BER) performance of cooperative PD-NOMA in downlink VLC systems and to compare it to non-cooperative PD-NOMA under perfect channel state information (CSI). In this context, we drive a closed-form expression for BER and provide Monte Carlo simulation results to validate the derived analytical BER. Moreover, we calculate the achievable rate of each user for non-cooperative and cooperative PD-NOMA.

The remainder of this paper is organized as follows; in Section 2 we give an indoor downlink VLC channel description and system model explanation of non-cooperative and cooperative PD-NOMA. In Section 3, we analyze the BER system performance under perfect CSI. In Section 4, we provide numerical results and related discussions. Finally, we conclude the paper in Section 5.

\section{System Model}

We consider an indoor VLC system that consists of a single LED transmitter located on the ceiling of a room. This LED is used for illumination and communication simultaneously to serve $M$ users. We apply OOK modulation technique to modulate the light intensity based on the data received through a power line communications (PLC) network. Moreover, each user has a single photodetector (PD) facing the ceiling.

\subsection{VLC Channel Model}

Modeling the VLC channel in this paper, as shown in Figure 1, takes into account only the line of sight (LOS). Although VLC channel has a diffusive component, it can be neglected because the 
reflected signal has very low energy compared to that of the LOS signal [17]. The direct current (DC) channel gain between user $U_{k}$ and the LED is given as in [18] by:

$$
h_{k}=\left\{\begin{array}{lc}
\frac{A_{k}}{d_{k}^{2}} R_{o}\left(\phi_{k}\right) T_{s}\left(\psi_{k}\right) g\left(\psi_{k}\right) \cos \left(\psi_{k}\right), & 0 \leq \psi_{k} \leq \psi_{c} \\
0, & \psi_{k}>\psi_{c}
\end{array}\right.
$$

where $k=1,2, \ldots, M, A_{k}$ denotes the detection area of the $\mathrm{PD}, d_{k}$ donates the Euclidean distance between the LED and the $k$-th user. The PD field of view (FOV) is donated by $\psi_{c}$ while the angle of incidence with respect to the axis normal to the transmitter surface and the angle of irradiance with respect to the axis normal to the receiver surface are denoted by $\psi_{k}$ and $\phi_{k}$, respectively. $T_{s}\left(\psi_{k}\right)$ represents the gain of the optical filter used at the receiver and $g\left(\psi_{k}\right)$ is the gain of the optical concentrator, which is given as in [18] by:

$$
g\left(\psi_{k}\right)=\left\{\begin{array}{lc}
\frac{n^{2}}{\sin ^{2}\left(\Psi_{c}\right)}, & 0 \leq \psi_{k} \leq \psi_{c} \\
0, & \psi_{k}>\psi_{c}
\end{array}\right.
$$

where $n$ is the corresponding reflective index of the optical concentrator. Furthermore, $R_{o}\left(\phi_{k}\right)$ is the Lambertian radiant intensity of the LEDs, represented as:

$$
R_{o}\left(\phi_{k}\right)=\frac{m+1}{2 \pi} \cos ^{m}\left(\phi_{k}\right)
$$

where $m$ is the order of Lambertian emission defined as:

$$
m=-\frac{\ln (2)}{\ln \left(\cos \left(\phi_{1 / 2}\right)\right)}
$$

where $\phi_{1 / 2}$ is the semi-angle at half power of an LED. The noise generated at the PDs has Gaussian distribution of zero mean and variance:

$$
\sigma_{n_{k}}^{2}=\sigma_{s h_{k}}^{2}+\sigma_{t h_{k}}^{2}
$$

where $\sigma_{s h_{k}}^{2}$ and $\sigma_{t h}^{2}$ are the variances of the shot noise and thermal noise, respectively. The shot noise variance at the $k$-th receiver is determined as in [19] by:

$$
\sigma_{s h_{k}}^{2}=2 q R_{p} P_{r_{k}} B+2 q I_{b g} I_{2}
$$

where $q$ is the electronic charge, $R_{p}$ is the photodetector responsivity, $P_{r_{k}}$ is the received optical power for user $k, B$ is the equivalent bandwidth, $I_{b g}$ is the photocurrent due to background radiation and $I_{2}$ is the noise bandwidth factor.

The thermal noise is generated within the transimpedance receiver circuit. If the noise effect from the gate leakage current is neglected, the thermal variance is expressed as in [19] by:

$$
\sigma_{t h_{k}}^{2}=\frac{8 \pi \kappa \mathrm{T}_{\mathrm{k}}}{G_{o l}} C_{p d} A I_{2} B^{2}+\frac{16 \pi^{2} k T_{k} \Gamma}{g_{m}} C_{p d}^{2} A^{2} I_{3} B^{3}
$$

which consists of feedback-resistor noise, and FET channel noise, where $k$ is the Boltzmann's constant, $T_{k}$ is absolute temperature, $G_{o l}$ is the open-loop voltage gain, $C_{p d}$ is the fixed capacitance of photodetector per unit area, $\Gamma$ is the FET channel noise factor, $g_{m}$ is the FET transconductance and $I_{3}=0.0868$ is a weighting function that depends on the input optical pulse shape [19]. 


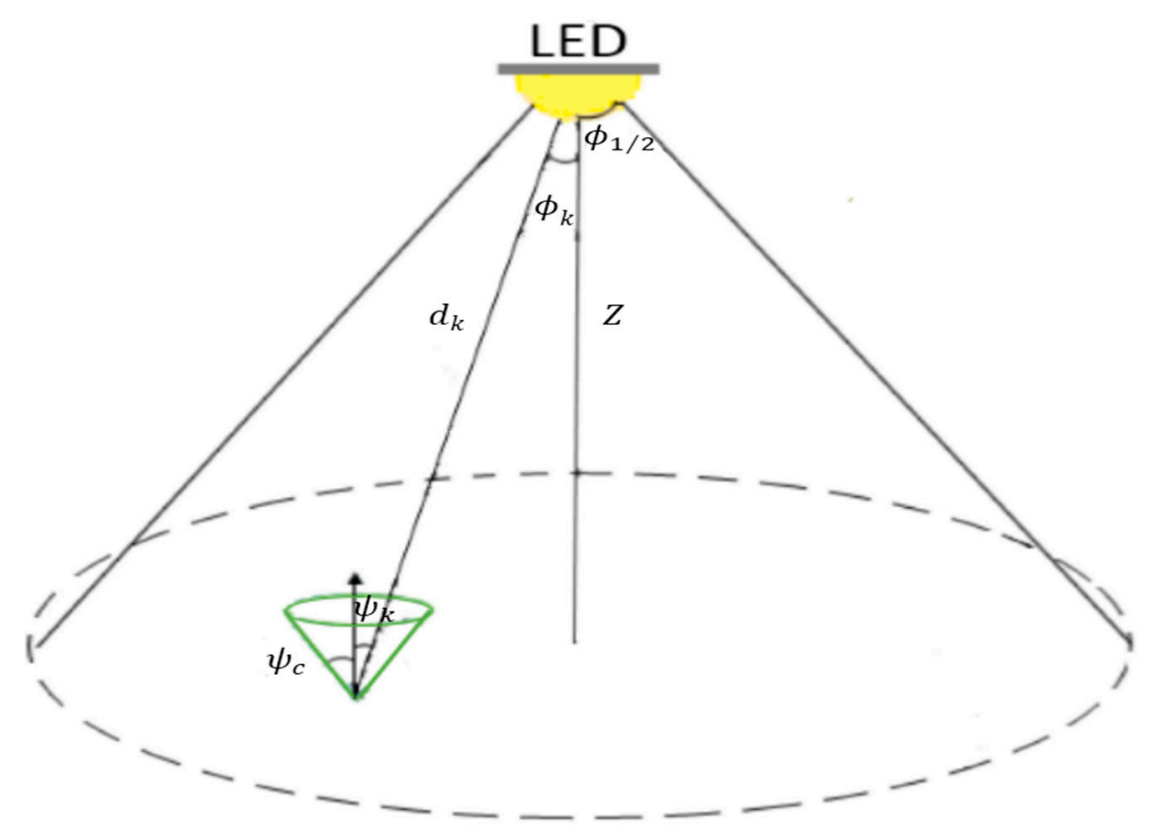

Figure 1. Visible light communication (VLC) system model.

\subsection{PD-NOMA Transmission}

The basic principle of PD-NOMA is to simultaneously serve multiple users over the same spectrum resources (i.e., time, frequency, and code) but with different power levels. For the considered system model and without loss of generality, assume that all of the users $U_{1}, \ldots, U_{M}$ are ordered based on their channel gains:

$$
h_{1} \leq \cdots \leq h_{k} \leq \cdots \leq h_{M}
$$

Based on PD-NOMA concept, signals for all users are superposed in the power domain. Thus, a DC bias added to ensure that the transmitted signal at the LED consists of $M$ non-negative signals $s_{1}$, $\ldots, s_{M}$ and is expressed as in [11] by:

$$
x=\sum_{l=1}^{M} a_{l} \sqrt{P_{\text {elec }}} s_{l}+I_{D C}
$$

where $a_{l}$ represents the power allocation coefficient for the $l$-th user, $P_{\text {elec }}$ represents the total electrical power, $s_{l}$ is the message intended for the $l$-th user, and $I_{D C}$ is the DC bias. Each message is assumed to have unity variance and zero mean. The use of PD-NOMA implies that $a_{1} \geq \cdots \geq a_{M}$ and power allocation coefficients should satisfy [11]

$$
\sum_{l=1}^{M} a_{l}^{2}=1 .
$$

The LED optical power is directly proportional to the LED driving current, the optical power can be expressed as:

$$
P_{o p t}=E[x]=I_{D C}
$$

where $E[$.$] denotes the statistical expectation. The received signal at the k$-th user is given by:

$$
y_{k}=\sqrt{P_{\text {elec }}} h_{k} \sum_{l=1}^{M} a_{l} s_{l}+n_{k}
$$


where $n_{k}$ denotes the real-valued additive white Gaussian noise (AWGN) with zero mean and variance $\sigma_{n}^{2}$.

\subsection{Cooperative PD-NOMA}

Consider a system model with the main light source at the ceiling and $M$ users. Cooperative PD-NOMA concept is divided into two phases: the direct transmission phase and the cooperative phase.

\subsubsection{The Direct Transmission Phase}

As already mentioned in the PD-NOMA transmission, the LED source sends $M$ signals to the users based on the PD-NOMA concept as shown in Figure 2.

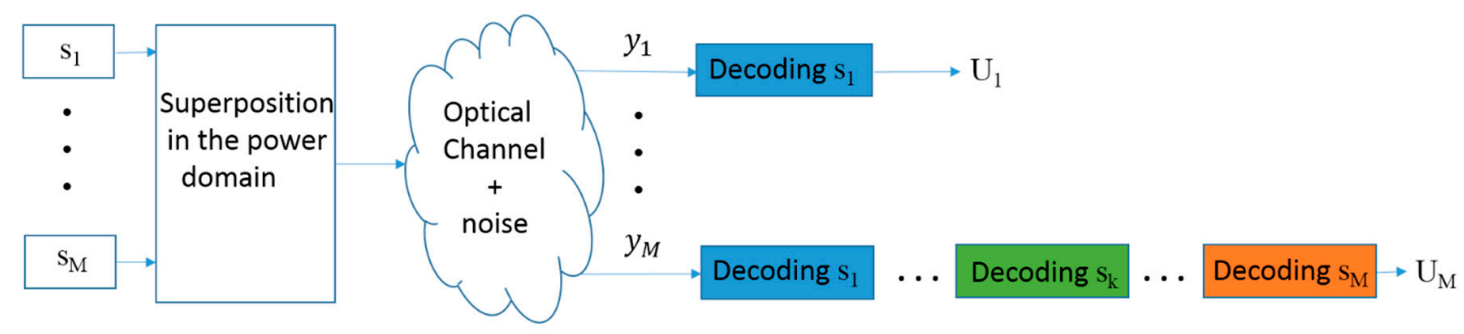

Figure 2. Power domain non-orthogonal multiple access (PD-NOMA)-VLC concept.

The $k$ receiver applies SIC to the $k$-th user, this removes the signal of the other users with higher power allocation. The received electrical power is expressed as:

$$
P_{r e_{k}}=P_{\text {elec }} h_{k} a_{k}^{2} .
$$

The received signal to interference plus noise ratio (SINR) for the $M$-th user to decode the $k$-th user's signal for $k<M$ is represented as in [16] by:

$$
\operatorname{SINR}_{M, k}=\frac{\left(h_{M} a_{k}\right)^{2}}{\sum_{l=k+1}^{M}\left(h_{M} a_{l}\right)^{2}+\frac{1}{\rho_{k}}}
$$

where $\rho_{k}=\frac{P_{\text {elec }}}{\sigma_{n_{k}}^{2}}$ represents the transmitted SNR. After the $M$-th user decodes and subtracts other users' signals, its own message is decoded with $\operatorname{SINR}_{M, M}=\rho\left(h_{M} a_{M}\right)^{2}$. The achievable data rate for the $k$-th user is given as in [11] by:

$$
R_{k}=\left\{\begin{array}{lc}
\frac{1}{2} \log _{2}\left(1+\frac{\left(h_{k} a_{k}\right)^{2}}{\sum_{l=k+1}^{M}\left(h_{k} a_{l}\right)^{2}+\frac{1}{\rho_{k}}}\right), & k=1, \cdots, M-1, . \\
\frac{1}{2} \log _{2}\left(1+\rho_{k}\left(h_{k} a_{k}\right)^{2}\right), & k=M
\end{array} .\right.
$$

The above equation is conditioned that the $k$-th user has to successfully detect the signal for all lower order users by satisfying the following condition:

$$
R_{k \rightarrow j}= \begin{cases}\frac{1}{2} \log _{2}\left(1+\frac{\left(h_{k} a_{j}\right)^{2}}{\sum_{l=j+1}^{M}\left(h_{k} a_{l}\right)^{2}+\frac{1}{\rho_{k}}}\right) \geq \widetilde{R}_{j,} & j \leq k, j \neq M \\ \frac{1}{2} \log _{2}\left(1+\rho_{k}\left(h_{k} a_{j}\right)^{2}\right) \geq \widetilde{R}_{j}, & j=k=M\end{cases}
$$

where $R_{k \rightarrow j}$ is the rate that the $k$-th user needs to detect the message signal for the $j$-th user and $\widetilde{R}_{j}$ is the targeted data rate for the $j$-th user to successfully detect its own message signal. 


\subsubsection{Cooperative Phase}

In this phase, the users cooperate with each other to improve the link reliability as shown in Figure 3 by dedicating a certain time slot for each user that acts as a relay. All users located far away from the main LED have another PD facing them that acts a relay. While all users located near the main LED source that act as relays are equipped with an LED to transmit in the cooperative phase. We need $(M-1)$ PDs and $(M-1)$ LEDs to achieve user cooperation, exactly similar in the specification as the main LED and PDs used in the direct transmission phase.

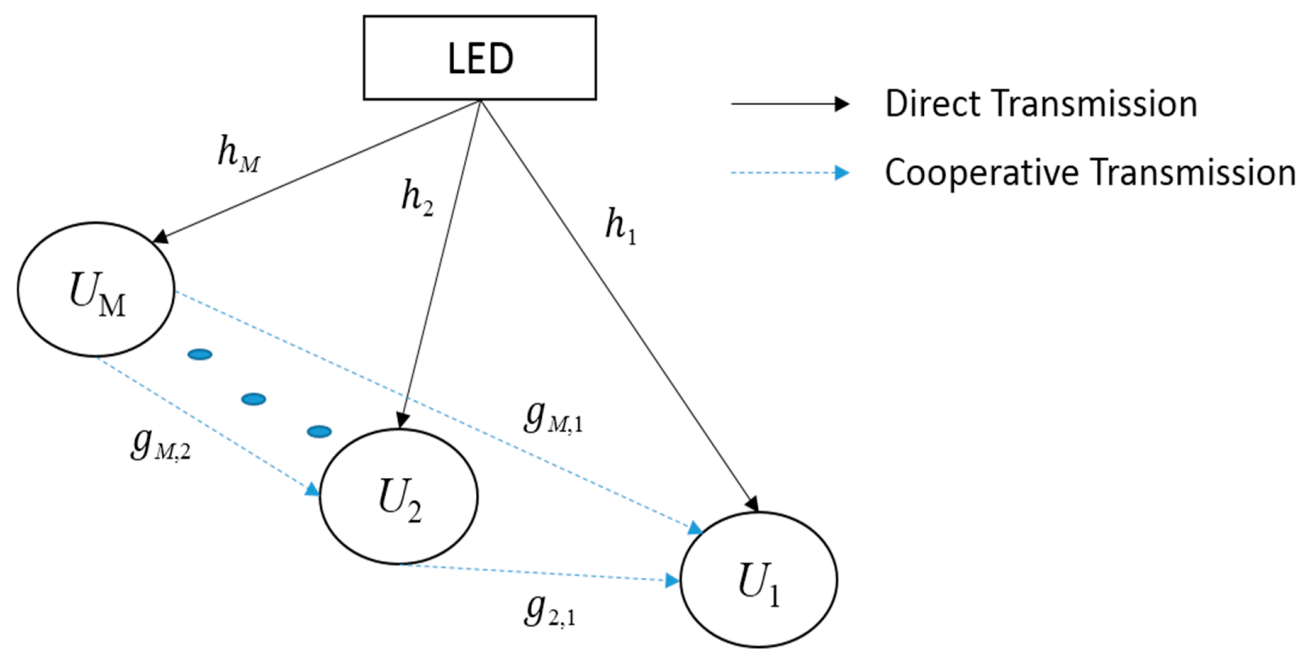

Figure 3. Cooperative PD-NOMA system model.

The cooperative VLC channel model in the case of two users is shown in Figure 4. The DC channel gain between $U_{2}$ and $U_{1}$ is expressed as:

$$
g_{2,1}=\left\{\begin{array}{lc}
\frac{A_{k}}{d_{2,1}^{2}} R_{o}\left(\phi_{2,1}\right) T_{s}\left(\psi_{2,1}\right) g\left(\psi_{2,1}\right) \cos \left(\psi_{2,1}\right), & 0 \leq \psi_{2,1} \leq \psi_{c} \\
0, & \psi_{2,1}>\psi_{c}
\end{array}\right.
$$

where $d_{2,1}$ donates the Euclidean distance between $U_{2}$ and $U_{1}$. The angle of incidence with respect to the axis normal to the $U_{2}$ LED surface and the angle of irradiance with respect to the axis normal to the $U_{1}$ PD surface are denoted by $\psi_{2,1}$ and $\phi_{2,1}$, respectively.

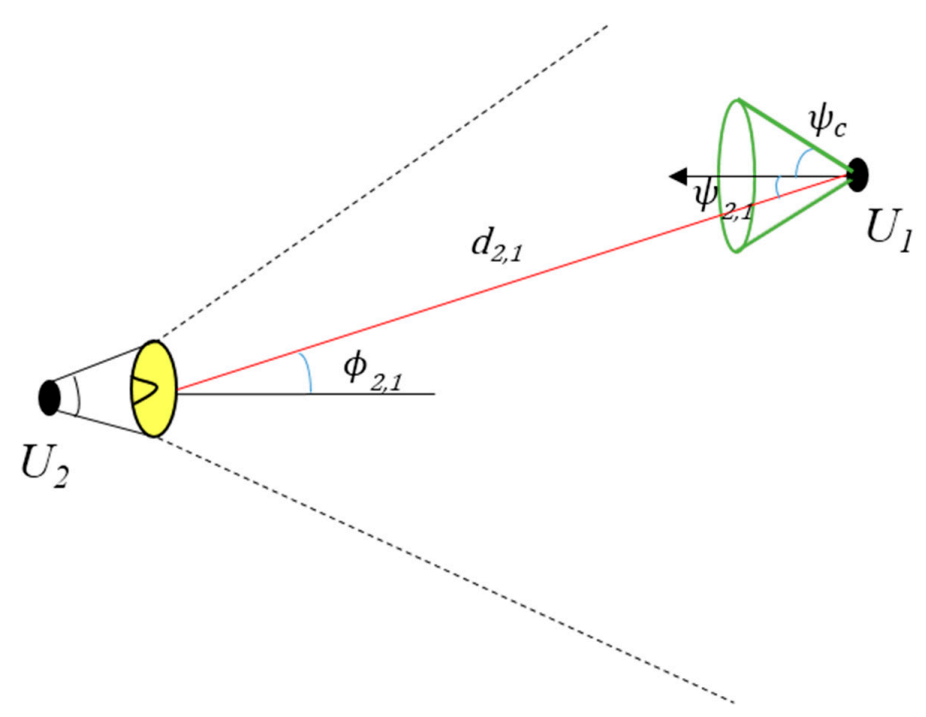

Figure 4. Cooperative VLC system model with two users. 
During the cooperative phase, $(M-1)$ time slots are needed where each user has a dedicated time slot to transmit, except for the user farthest from the main LED. Using time slots allows all users except the nearest to the main LED to take advantage of the cooperative phase. Otherwise, the farthest user can only improve its signal reception in the case of using only one time slot in the cooperative phase. The $M$-th user transmits in the first time slot a combination of $(M-1)$ messages with power allocation coefficients $q_{M}$, where $\sum_{l=1}^{M-1} q_{M, l}^{2}=1$. The received signal at the $k$-th user for $k<M$ is given as in [16] by:

$$
y_{2, k}=\sum_{l=1}^{M-1} \sqrt{P_{\text {elec }}} g_{M, k} q_{M, l} s_{l}+n_{2, k}
$$

where $g_{M, k}$ denotes the channel gain between user $M$ and the $k$-th user. Maximum ratio combining (MRC) is used to combine the received signal from both phases as shown in Table 1.

Table 1. Time slot analysis of the cooperative phase.

\begin{tabular}{|c|c|c|c|}
\hline Time Slot & & Cooperative Phase Process & \\
\hline 1 & $\begin{array}{l}U_{M} \text { transmits messages } \\
\text { intended for } U_{M-1} \\
\text { through } U_{1} \text { using the } \\
\text { PD-NOMA concept. }\end{array}$ & $\begin{array}{l}U_{M-1} \text { combines the received } \\
\text { signals from light-emitting } \\
\text { diode and } U_{M} \text { using the } \\
\text { maximum ratio } \\
\text { combining technique. }\end{array}$ & $\begin{array}{l}U_{M-1} \text { decodes the maximum } \\
\text { ratio combining signal to } \\
\text { extract } U_{1}, U_{2}, \ldots, U_{M-1} \\
\text { messages using successive } \\
\text { interference cancellation. }\end{array}$ \\
\hline 2 & $\begin{array}{l}U_{M-1} \text { transmits } \\
\text { messages intended for } \\
U_{M-2} \text { through } U_{1} \text { using } \\
\text { the PD-NOMA concept. }\end{array}$ & $\begin{array}{l}U_{M-2} \text { combines the received } \\
\text { signals from light-emitting } \\
\text { diode, } U_{M} \text { and } U_{M-1} \text {, using } \\
\text { the maximum ratio } \\
\text { combining technique. }\end{array}$ & $\begin{array}{l}U_{M-2} \text { decodes the maximum } \\
\text { ratio combining signal to } \\
\text { extract } U_{1}, U_{2}, \ldots, U_{M-2} \\
\text { messages using successive } \\
\text { interference cancellation. }\end{array}$ \\
\hline$\because$ & $\bullet$ & $:$ & $:$ \\
\hline$M-1$ & $\begin{array}{l}U_{2} \text { transmits message } \\
\text { intended for } U_{1} .\end{array}$ & $\begin{array}{l}U_{1} \text { combines the received } \\
\text { signals form light-emitting } \\
\text { diode, } U_{M}, \ldots, U_{2} \text {, using } \\
\text { maximum ratio } \\
\text { combining technique. }\end{array}$ & $U_{1}$ decodes its own message. \\
\hline
\end{tabular}

We should take into consideration that increasing the number of users leads to increasing the number of time slots used in the cooperative phase which decreasing the transmission rate. Therefore, if the number of users exceeds the limitation of the system, communication between users in a realistic scenario may be lost.

The SINR for the $(M-1)$-th user to decode the $k$-th user's message signal for $k<(M-1)$ is expressed as in [16] by:

$$
\operatorname{SINR}_{M-1, k}=\frac{\left(h_{M-1} a_{k}\right)^{2}}{\left(h_{M-1}\right)^{2} \sum_{l=k+1}^{M} a_{l}^{2}+\frac{1}{\rho_{k}}}+\frac{\left(g_{M, M-1} q_{M, k}\right)^{2}}{\left(g_{M, M-1}\right)^{2} \sum_{l=k+1}^{M-1} q_{M, l}^{2}+\frac{1}{\rho_{k}}} .
$$

The SINR for the $(M-1)$-th user to decode its own message is expressed as:

$$
\operatorname{SINR}_{M-1, M-1}=\frac{\left(h_{M-1} a_{M-1}\right)^{2}}{\left(h_{M-1} a_{M}\right)^{2}+\frac{1}{\rho_{k}}}+\rho_{k}\left(g_{M, M-1} q_{M, M-1}\right)^{2} .
$$


The $(M-i+1)$-th user transmits in the $i$-th time slot a combination of $(M-i)$ messages where $1 \leq$ $i \leq(M-1)$, with power allocation coefficients $q_{M-i+1, l}$, where $\sum_{l=1}^{M-i} q_{M-i+1, l}^{2}=1$. The received signal at the $k$-th user for $k<(M-i+1)$ is given by:

$$
y_{2, k}=\sum_{l=1}^{M-i} \sqrt{P_{\text {elec }}} g_{M-i+1, k} q_{M-i+1, l} s_{l}+n_{i+1, k}
$$

The SINR for the $(M-i)$-th user to decode the $k$-th user's message signal for $1 \leq k<(M-i)$ is expressed as in [16] by:

$$
\operatorname{SINR}_{M-i, k}=\frac{\left(h_{M-i} a_{k}\right)^{2}}{\left(h_{M-i}\right)^{2} \sum_{l=k+1}^{M} a_{l}^{2}+\frac{1}{\rho_{k}}}+\sum_{j=1}^{i} \frac{\left(g_{M-j+1, M-i} q_{M-j+1, k}\right)^{2}}{\left(g_{M-j+1, M-i}\right)^{2} \sum_{l=k+1}^{M-j} q_{M-j+1, l}^{2}+\frac{1}{\rho_{k}}} .
$$

The SINR for the $(M-i)$-th to decode its own message is expressed as:

$$
\begin{gathered}
\operatorname{SINR}_{M-i, M-i}=\frac{\left(h_{M-i} a_{M-i}\right)^{2}}{\left(h_{M-i}\right)^{2} \sum_{l=M-i+1}^{M} a_{l}^{2}+\frac{1}{\rho_{k}}}+\sum_{j=1}^{i-1} \frac{\left(g_{M-j+1, M-i} q_{M-j+1, M-i}\right)^{2}}{\left(g_{M-j+1, M-i}\right)^{2} \sum_{l=M-i+1}^{M-j} q_{M-j+1, l}^{2}+\frac{1}{\rho_{k}}} \\
+\rho_{k}\left(g_{M-i+1, M-i} q_{M-i+1, M-i}\right)^{2} .
\end{gathered}
$$

Finally, we can see from Equation (23) that the second and third terms represent the effect of cooperative PD-NOMA for enhancing the link reliability. These two terms are responsible for increasing the $k$-th user's SINR.

\section{BER Analytical Expression}

In this section, we discuss the equations used for calculating the BER of non-cooperative and cooperative PD-NOMA.

\subsection{Non-Cooperative PD-NOMA}

Since OOK modulation is considered, the signal constellation of $U_{k}$ is represented as $\sum_{i=1}^{2^{M-k}} \sum_{j=k+1}^{M} P_{j} V_{i j}$, where the matrix $V$ exhibits the possible position of the symbols in the constellation based on the transmitted OOK message $S_{k}$. Figure 5 shows a signal constellation example for three users.

$$
V=\left[\begin{array}{ccccc}
V_{1 k} & V_{1 k+1} & \cdots & V_{1 M-1} & V_{1 M} \\
V_{2 k} & V_{2 k+1} & \cdots & V_{2 M-1} & V_{2 M} \\
V_{3 k} & V_{3 k+1} & \cdots & V_{3 M-1} & V_{3 M} \\
\vdots & \vdots & \vdots & \vdots & \vdots \\
V_{2^{M-k} k} & V_{2^{M-k} k+1} & \cdots & V_{2^{M-k} M-1} & V_{2^{M-k} M}
\end{array}\right]=\left[\begin{array}{ccccc}
0 & 0 & \cdots & 0 & 0 \\
0 & 0 & \cdots & 0 & 1 \\
0 & 0 & \cdots & 1 & 0 \\
\vdots & \vdots & \vdots & \vdots & \vdots \\
1 & 1 & \cdots & 1 & 1
\end{array}\right] .
$$

Probability of error in decoding the $k$-th signal is expressed as:

$$
P_{e_{k}}=1-\left[\frac{1}{2^{M-k}} \sum_{i=1}^{2^{M-k}} 1-Q\left(\frac{\sqrt{2} R_{p} h_{k}\left(T h_{k}-\sum_{j=k+1}^{M} P_{j} V_{i j}\right.}{\sqrt{\sigma_{n_{k}}^{2}}}\right)\right]
$$

where $T h_{k}$ is the maximum likelihood (ML) detection threshold of $U_{k}$, which is expressed as:

$$
T h_{k}=\frac{\sum_{m=k}^{M} P_{m}}{2} .
$$


The Gaussian $Q$ function is given by:

$$
Q(x)=\frac{1}{\sqrt{2 \pi}} \int_{x}^{\infty} \exp \left(\frac{-\mathrm{y}^{2}}{2}\right) d y .
$$

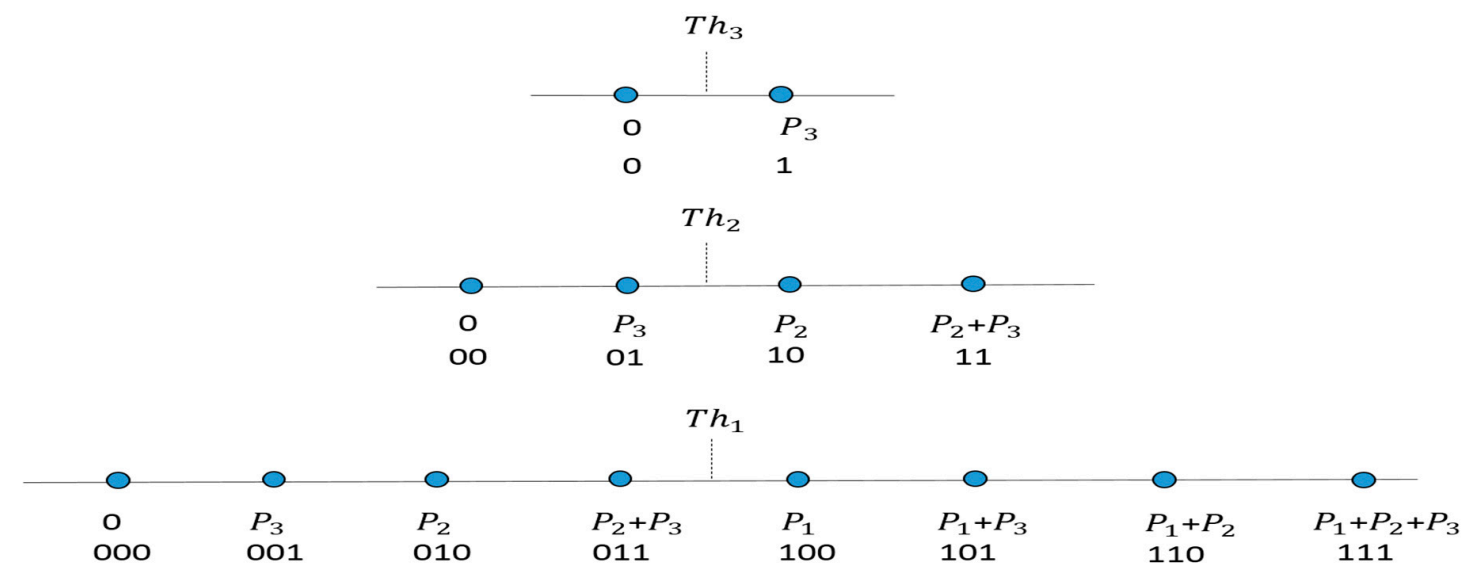

Figure 5. Signal constellation for three users.

\subsection{Cooperative PD-NOMA}

\subsubsection{Single Relay}

In the case of a single relay (one branch, two users), as shown in Figure 6, errors at $U_{1}$ occur either when $U_{2}$ receives in error the transmitted signal through the LED- $U_{2}$ link, and $U_{1}$ receives correctly the transmitted signal through $U_{2}-U_{1}$ link; or when LED- $U_{2}$ transmitted signal is received correctly, and $U_{2}-U_{1}$ transmitted signal is received in error. Hence, the LED $-U_{2}-U_{1}$ link has error probability given by:

$$
P_{e_{\mathrm{LED}}-U_{2}-u_{1}}=\left[1-P_{e_{U_{2}}-u_{1}}\right] P_{e_{\mathrm{LED}}-U_{2}}+\left[1-P_{e_{\mathrm{LED}}-U_{2}}\right] P_{e_{U_{2}}-u_{1}}
$$

where $P_{e_{\mathrm{LED}}-U_{2}}$ and $P_{e_{U_{2}}-u_{1}}$ are the error probabilities of LED- $U_{2}$ link and $U_{2}-U_{1}$ link, respectively.

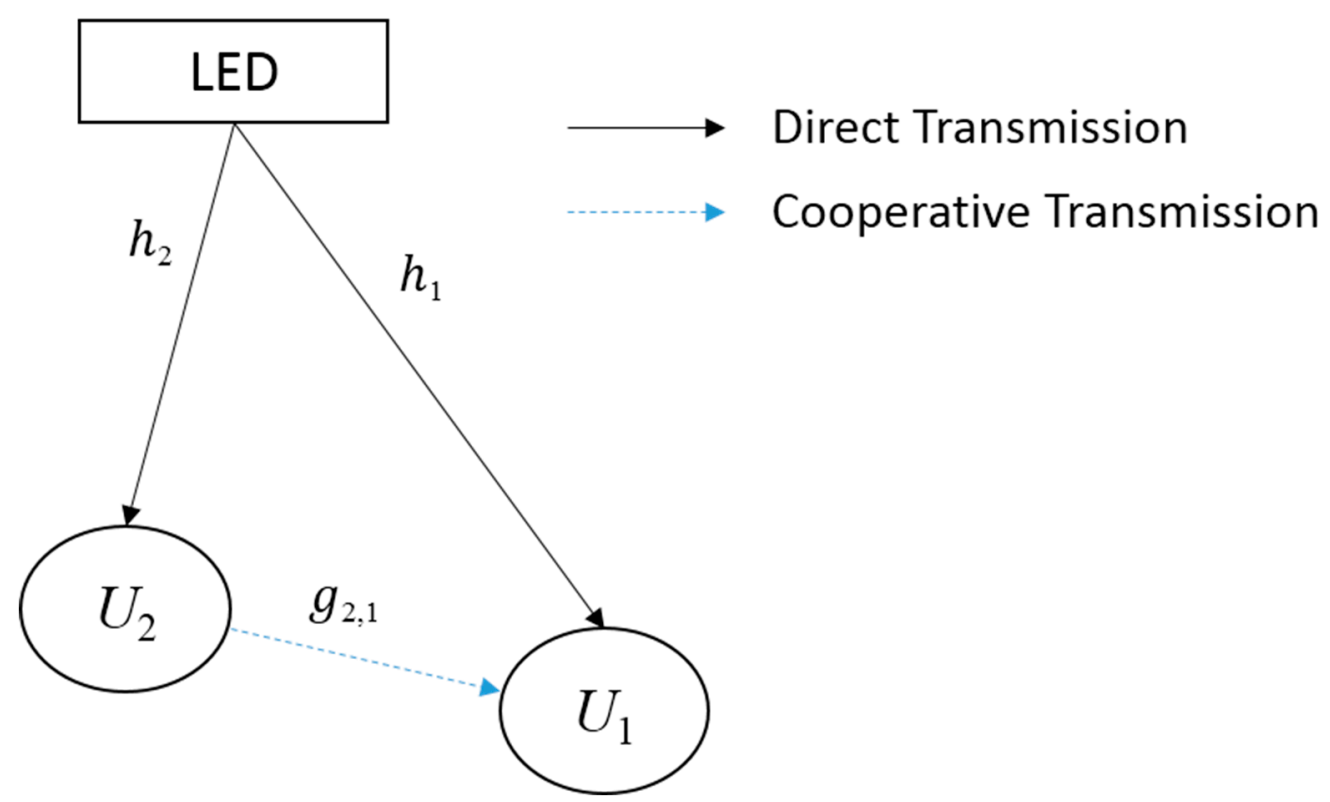

Figure 6. Cooperative PD-NOMA system model for two users. 
After combining the received signals from the direct transmission and $U_{1}$ using MRC, the probability of error of $U_{1}$ is expressed in consistence with the presented model as in [20] by:

$$
P_{e_{1}}=\left[1-P_{e_{\mathrm{LED}}-U_{2}}\right] Q\left(\frac{\sqrt{2}\left(S N R_{\mathrm{LED}}-u_{1}+S N R_{e q}\right)}{\sqrt{S N R_{\mathrm{LED}}-u_{1}+\frac{S N R_{e q} q^{2}}{S N R_{L_{2}}-u_{1}}}}\right)+P_{e_{\mathrm{LED}}-U_{2}} Q\left(\frac{\sqrt{2}\left(S N R_{\mathrm{LED}}-u_{1}-S N R_{e q}\right)}{\sqrt{S N R_{\mathrm{LED}}-u_{1}+\frac{S N R_{e q}{ }^{2}}{S N R U_{2}-u_{1}}}}\right) .
$$

The equivalent SNR for the link LED- $U_{2}-U_{1}$ is represented as in [13] by

$$
S N R_{e q}=\frac{S N R_{\mathrm{LED}}-\mathrm{U}_{2} S N R_{U_{2}}-U_{1}}{S N R_{\mathrm{LED}}-U_{2}+S N R_{U_{2}}-U_{1}+1}
$$

where

$$
\mathrm{SNR} R_{\mathrm{LED}}-U_{2}=\rho_{k}\left(h_{2} a_{2}\right)^{2}
$$

and

$$
\mathrm{SNR}_{U_{2}-U_{1}}=\rho_{k}\left(g_{2,1} q_{2,1}\right)^{2} .
$$

Substituting Equations (30)-(32) in Equation (29), we get the probability of error of $U_{1}$. However, we calculate the probability of error of $U_{2}$ using Equation (25).

\subsubsection{Multiple Relays}

We generalize our model to consider a cooperative system with $M-k+1$ branches $b_{0}, b_{1}, \ldots$, $b_{M-k+1}$ where $b_{0}$ represents the direct transmission for $1 \leq k<(M-1)$ and branch $b_{g}$ consists of $N_{g}$ relays. The probability of error of the user $k$ is expressed as:

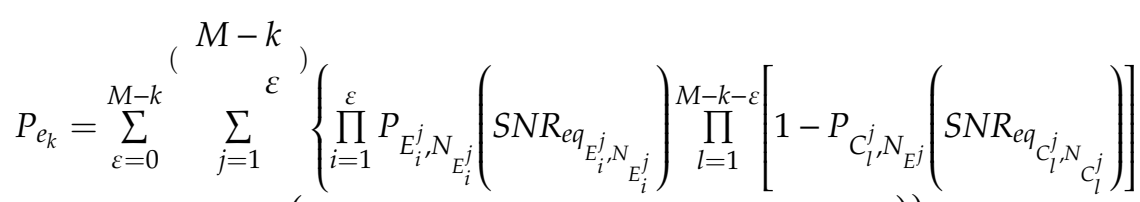

$$
\begin{aligned}
& \left.\times Q\left(\frac{\sqrt{2}\left(S N R_{L E D-k}+\sum_{l=1}^{M-k-\varepsilon} S N R_{e q} C_{l}^{j}-\sum_{i=1}^{\varepsilon} S N R_{e q} E_{i}^{j}\right.}{\sqrt{S N R_{L E D-k}+\sum_{g=1}^{M-k} \frac{S N R_{e q g}{ }^{2}}{S N R_{g, k}}}}\right)\right\}
\end{aligned}
$$

where $\varepsilon$ is used to index the users forwarding erroneously detected message to $U_{\mathrm{k}}, E^{j}$ and $C^{j}$ are set of $\varepsilon$ and $(M-k-\varepsilon)$ distinct elements from the set $\{1,2, \ldots, M-k\}$, respectively. $E_{i}^{j}$ and $C_{l}^{j}$ are the $i$-th and $l$-th elements of $E^{j}$ and $C^{j}$, respectively. It is noted here that $\varepsilon=0$ when all users are transmitting the message intended to $U_{\mathrm{k}}$ correctly. In [20], the authors describe a system that consists of source, relays and destination with a probability of error always calculated at the destination. Therefore, we modify Equation (28) [20] into Equation (33) to be in consistence with the presented cooperative PD-NOMA system in Figure 3 where the users with good channel conditions are relays. For any number of relays, the probability of error is calculated at any user, not only at the destination, which is $U_{1}$ in the presented model. Without loss of generality, we calculate the equivalent SNR of each branch and the direct transmission SNR using the same concept as Equations (30) and (31), respectively.

\section{Numerical Results and Discussion}

In this section, we evaluate the performance of the proposed cooperative PD-NOMA-VLC downlink system. We provide Monte Carlo simulations to substantiate the accuracy of the derived BER analytical expressions. We investigate the BER and achievable rate performance based on the system model proposed in Section 2. A realistic indoor environment of a $4 \mathrm{~m} \times 4 \mathrm{~m} \times 3 \mathrm{~m}$ room is considered with the main LED source placed at the center of the ceiling with total optical power of 1 $\mathrm{W}$. We consider the same system parameter as in [21] where three users are assumed to be served via 
PD-NOMA simultaneously. We use a fixed power allocation (FPA) strategy where the power allocated to the $k$-th user is $a_{k}^{2}=\Delta a_{k-1}^{2}$ under the constraint $\sum_{l=1}^{M} a_{l}^{2}=1$, where $\Delta$ is the power allocation factor $(0<\Delta<1)$ and the best BER performance achieved at $\Delta=0.3$ as in [8]. We summarize the simulation parameters of the proposed scenario in Table 2, and show all fixed user locations, FOV and channel gains in Table 3.

Table 2. Simulation parameters "Reproduced with permission from [21], IEEE, 2017".

\begin{tabular}{ccc}
\hline Description & Symbol & Value \\
\hline LED semi-angle & $\phi_{1 / 2}$ & $50^{\circ}$ \\
PD FOV & $\psi_{c}$ & $45^{\circ}$ \\
Refractive index & $n$ & 1.5 \\
Optical filter gain & $T^{s}\left(\psi_{k}\right)$ & 1 \\
Responsivity & $R_{p}$ & $1 \mathrm{~A} / \mathrm{W}$ \\
PD detection area & $A_{k}$ & $1 \mathrm{~cm}^{2}$ \\
Bandwidth & $B$ & $10 \mathrm{MHz}$ \\
open-loop voltage gain & $C_{o l}$ & 10 \\
fixed capacitance of PD & $C_{p d}$ & $112 \mathrm{pF} / \mathrm{cm}^{2}$ \\
Absolute temperature & $T_{k}$ & $298 \mathrm{~K}$ \\
FET transconductance & $g_{m}$ & $30 \mathrm{mS}$ \\
Noise bandwidth factors & $I_{3}$ & 0.0868 \\
FET channel noise factor & $\Gamma$ & 1.5 \\
Background current & $I_{b g}$ & $5100 \mu \mathrm{A}$ \\
Noise bandwidth factors & $I_{2}$ & 0.562 \\
\hline
\end{tabular}

Table 3. Users' setup “Reproduced with permission from [21], IEEE, 2017”.

\begin{tabular}{cccc}
\hline User & Location & FOV & Channel Gain \\
\hline$U_{1}$ & $(2.5,2.5,1.25)$ & $60^{\circ}$ & $0.2835 \times 10^{-4}$ \\
$U_{2}$ & $(2.4,2.4,1.25)$ & $45^{\circ}$ & $0.4787 \times 10^{-4}$ \\
$U_{3}$ & $(2.3,2.3,1.25)$ & $45^{\circ}$ & $0.5272 \times 10^{-4}$ \\
\hline
\end{tabular}

Figure 7 demonstrates cooperative and non-cooperative PD-NOMA-VLC BER performance of the two users under perfect CSI. Although the far user (i.e., $U_{1}$ ) has the worst channel condition, it achieves better BER due to the high power allocated to its signal. Therefore, as the decoding order decreases the BER performance increases, which allows $U_{1}$ to have the best BER performance by decoding its own signal directly and treating others as noise. Since $U_{2}$ is the nearest to the main LED source it only has one $\mathrm{PD}$ to receive the transmitted signal during direct transmission phase. It decodes and subtracts the message intended for $U_{1}$ using SIC before decoding its own signal. Then in the cooperative phase, $U_{2}$ acts as a relay to retransmit the $U_{1}$ message. Therefore, there is no change in the BER performance of $U_{2}$ in non-cooperative and cooperative PD-NOMA. Moreover, $U_{1}$ receives $y_{1}$ from the LED transmitter and the ML decoded signal $y_{2,1}$ from $U_{2}$. MRC is used to combine the received signals from both direct transmission and cooperative phases. It can be seen that cooperative PD-NOMA improved $U_{1}$ BER performance by about $8.2 \mathrm{~dB}$ at $10^{-6}$ compared to non-cooperative. Figure 8 shows the BER of cooperative and non-cooperative PD-NOMA in a three-user scenario where $U_{2}$ and $U_{3}$ act as relays. In this scenario, $U_{3}$ is the nearest to the main LED source. It decodes and subtracts the messages intended for $U_{1}$ and $U_{2}$ using SIC before decoding its own signal. Therefore, in the direct transmission phase, $U_{2}$ receives $y_{2}$ from the main LED. While in the cooperative phase, it receives the ML decoded signal $y_{2,2}$ from $U_{3}$ in the first time slot. Similarly, $U_{1}$ receives signals from the LED transmitter, from $U_{3}$ during the first time slot, and from $U_{2}$ during the second time slot. MRC is used at $U_{2}$ and $U_{1}$ receivers to combine the signals from both phases. It is noted that the BER performance of $U_{3}$ is the same in both phases while the BERs of $U_{2}$ and $U_{1}$ improved by about $2 \mathrm{~dB}$ and $9 \mathrm{~dB}$ at $10^{-6}$, respectively. 


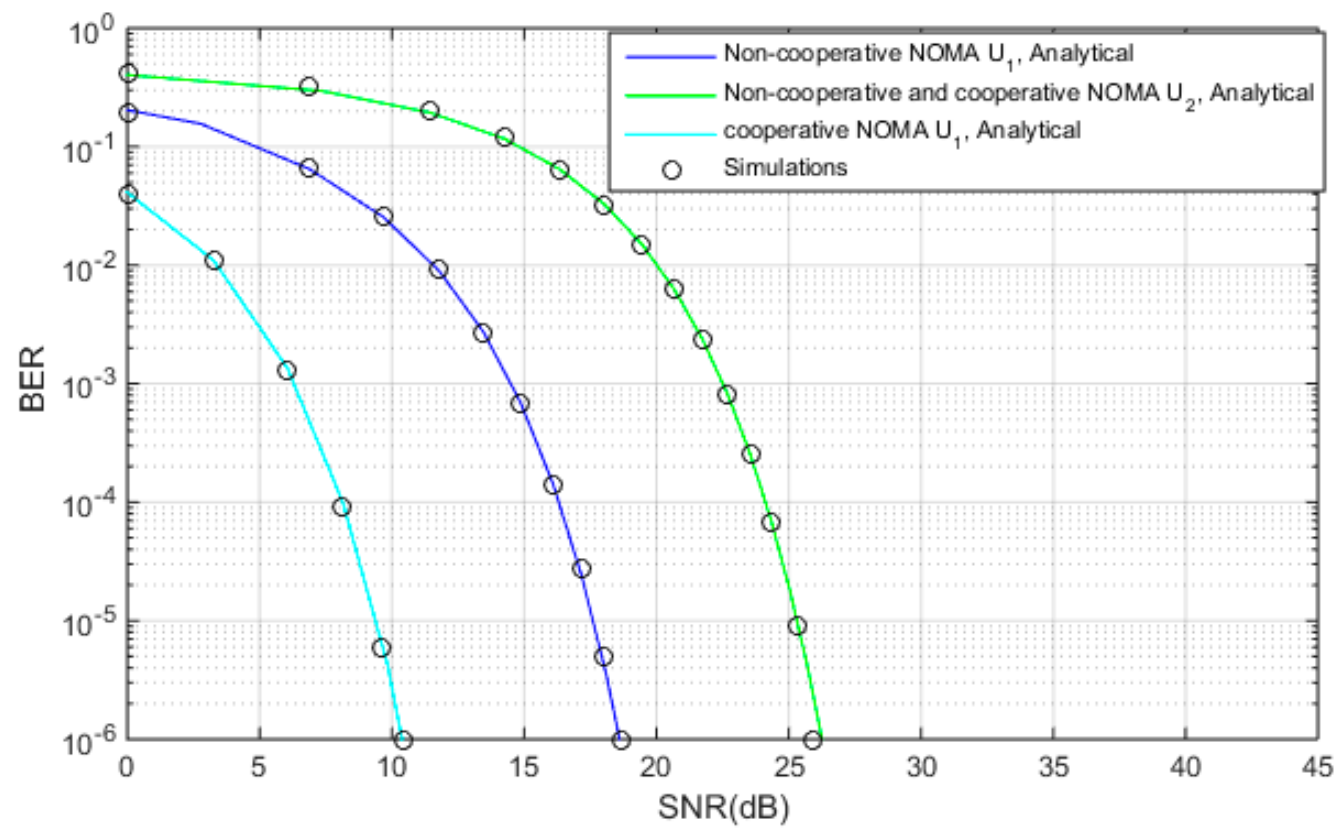

Figure 7. Bit error rate (BER) performance for cooperative PD-NOMA with two users.

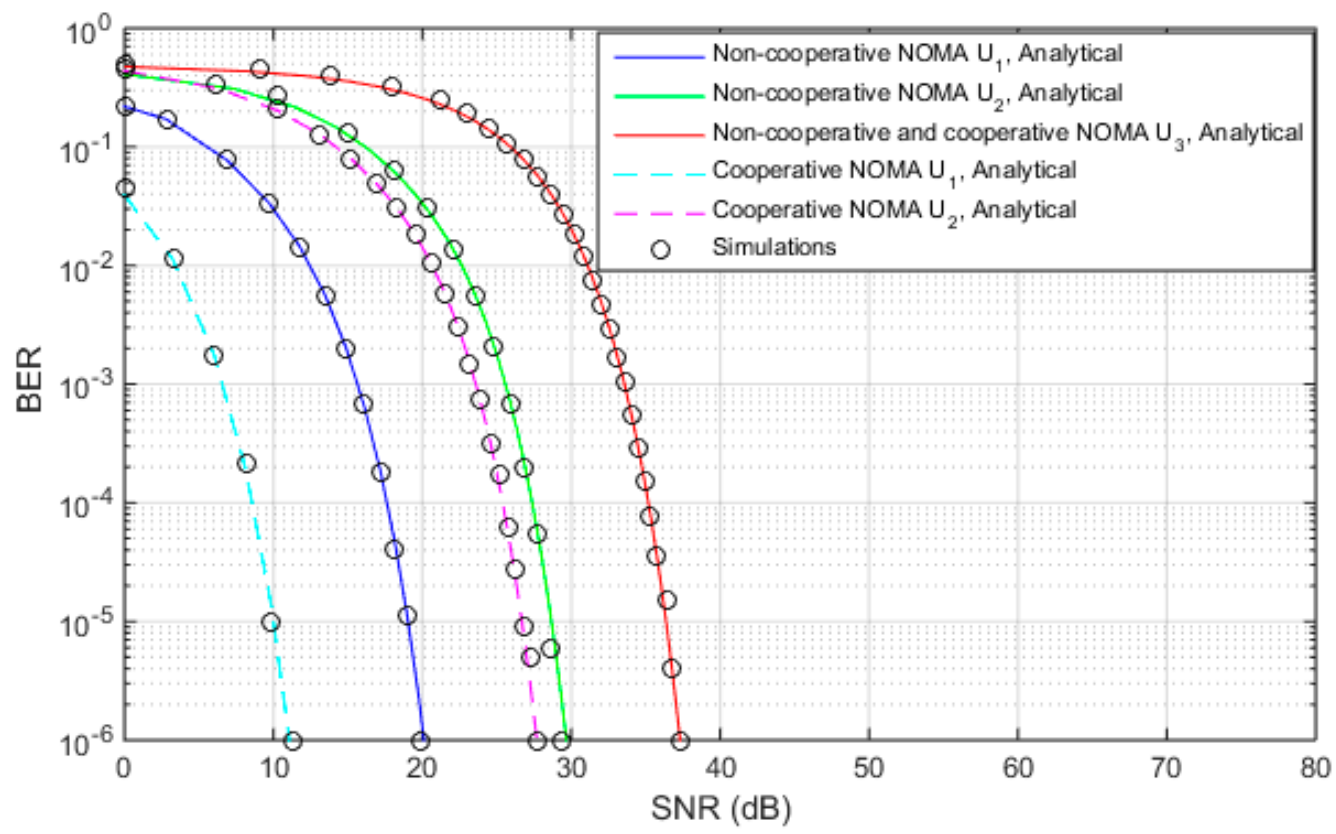

Figure 8. BER performance for cooperative PD-NOMA with three users.

Figure 9 shows the effect of decreasing the transmitted power of the users that act as relays on the BER performance of cooperative NOMA. This demonstrates a more realistic VLC scenario where the LEDs that are used as relays have one-fourth the power of the main LED. Although the transmitted power in the direct phase is still the same, the results show that the BER performance of the cooperative NOMA highly depends on the LED power of the relays. It is noted that the BER performances of $U_{2}$ and $U_{1}$ in the cooperative phase are degraded when compared to Figure 8 by $1.45 \mathrm{~dB}$ and $6.7 \mathrm{~dB}$ at $10^{-6}$, respectively. Also, another scenario can be considered without decreasing the power of the relays by adjusting the users at specific angles to exploit the non-LOS propagation of the signal. 


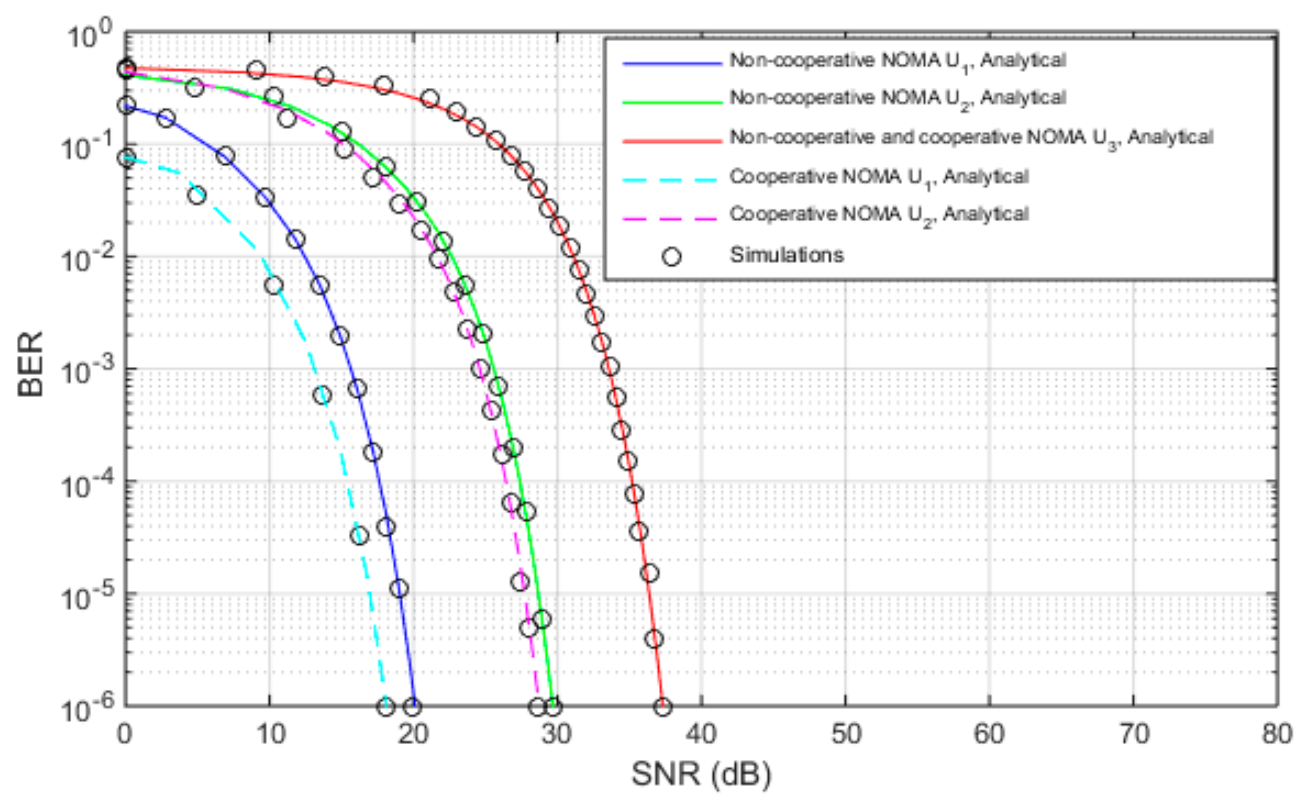

Figure 9. BER performance for cooperative PD-NOMA with relay power $0.25 \mathrm{~W}$.

Figure 10 shows the BER performance of cooperative NOMA with different users' setup and their coordinates are depicted in Table 4; Figures 8 and 10 have the same channel gain ordering in the direct transmission phase as in Equation (8) but with different channel gain ordering in the cooperative phase. Results show degradation in the BER performance of cooperative PD-NOMA for $U_{1}$ and $U_{2}$ when compared to Figure 8. The BER analytical expressions are matched with the Monte Carlo simulation results and are not limited to specific physical parameters.

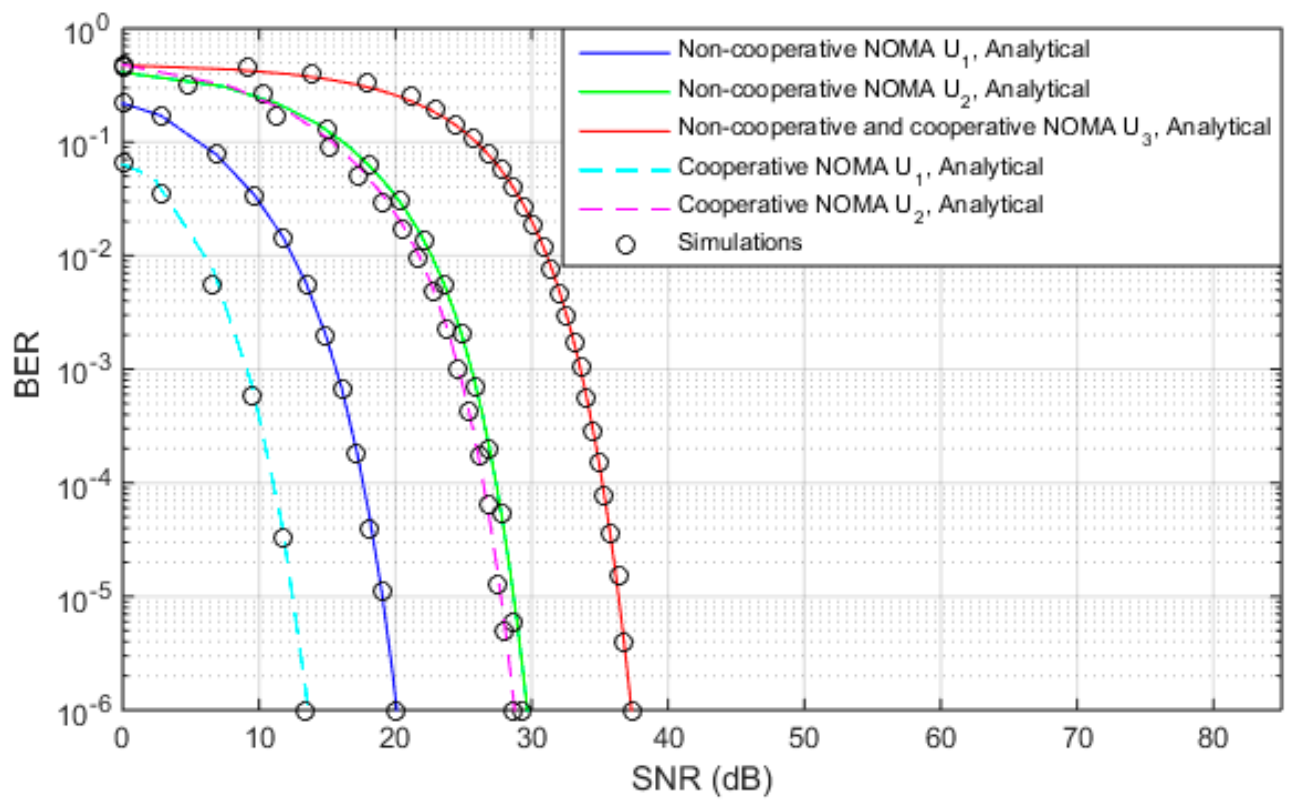

Figure 10. BER performance for cooperative PD-NOMA with different users' setup. 
Table 4. Users' location.

\begin{tabular}{cc}
\hline User & Location \\
\hline$U_{1}$ & $(2.9,1.9,1.25)$ \\
$U_{2}$ & $(1.5,1.7,1.25)$ \\
$U_{3}$ & $(2.2,2.5,1.25)$ \\
\hline
\end{tabular}

As shown in Figure 11, we evaluate the achievable rate in a two-user scenario. In non-cooperative PD-NOMA, $U_{2}$ has a higher achievable rate than $U_{1}$ owing to the ability of $U_{2}$ to cancel $U_{1}$ interference successfully by applying SIC. It is clear that using cooperative PD-NOMA dramatically improved $U_{1}$ achievable rate by $14.1 \mathrm{bit} / \mathrm{s} / \mathrm{Hz}$ at $45 \mathrm{~dB}$ due to increasing the SINR of $U_{1}$. Similarly, Figure 12 presents the achievable rate in a three-user scenario where $U_{3}$ and $U_{2}$ act as relays. $U_{3}$ has the highest achievable rate in non-cooperative PD-NOMA, where it is the nearest user to the LED and has the ability to successfully cancel $U_{2}$ and $U_{1}$ interference by applying SIC. Moreover, using cooperative PD-NOMA enhanced $U_{2}$ and $U_{1}$ achievable rates by $12.4 \mathrm{bit} / \mathrm{s} / \mathrm{Hz}$ and $14.3 \mathrm{bit} / \mathrm{s} / \mathrm{Hz}$ at $45 \mathrm{~dB}$, respectively.

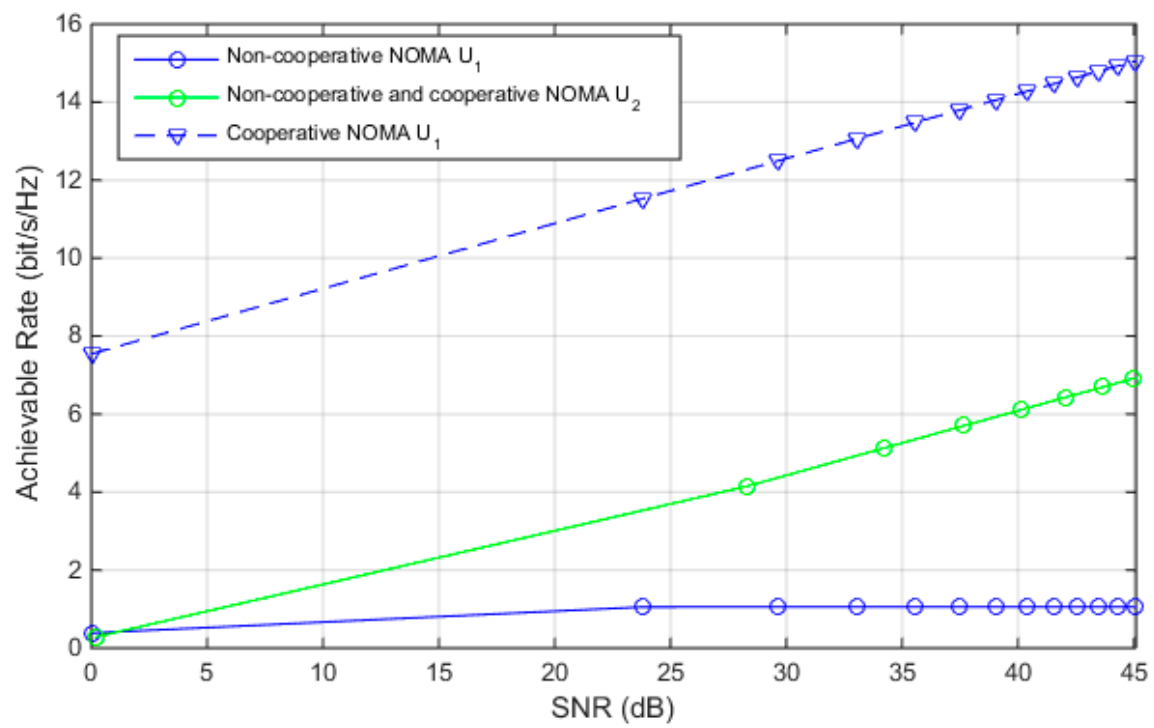

Figure 11. Achievable rate for cooperative PD-NOMA with two users.

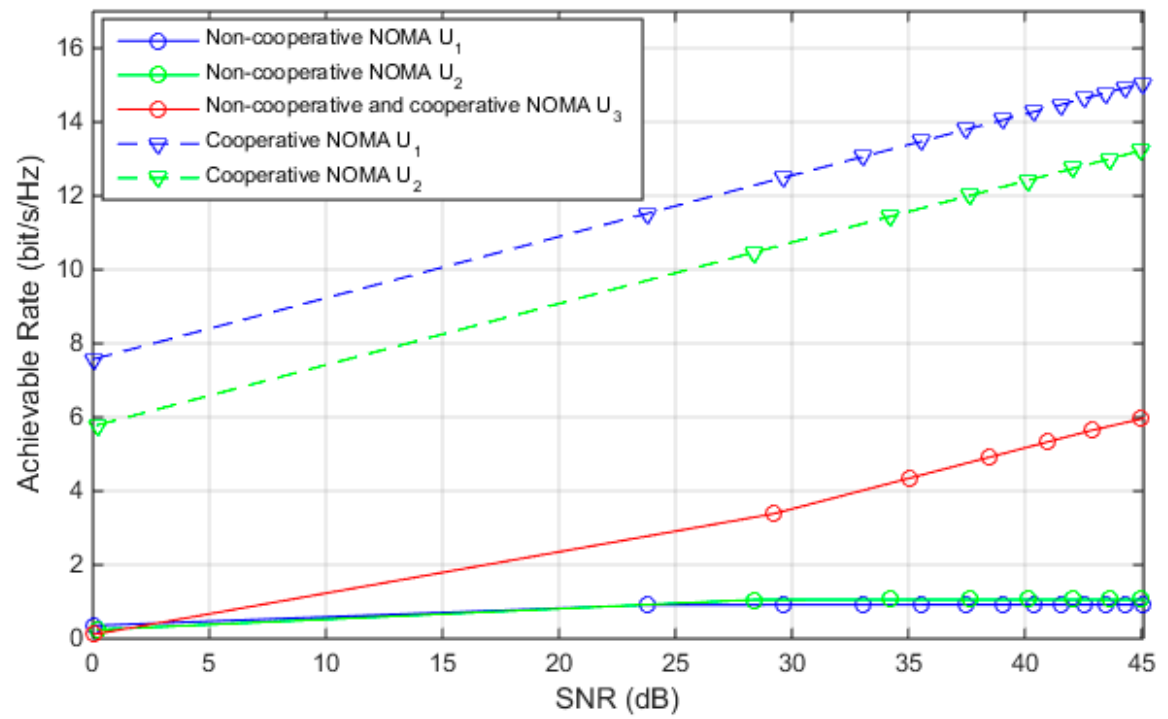

Figure 12. Achievable rate for cooperative PD-NOMA with three users. 


\section{Conclusions}

This paper investigates the performance of non-cooperative and cooperative PD-NOMA-VLC downlink systems under perfect CSI. BER analytical expressions are matched with the Monte Carlo simulation results. The obtained results show that the cooperative PD-NOMA enhanced $U_{1}$ BER performance by $8.2 \mathrm{~dB}$ at $10^{-6} \mathrm{BER}$ in a two-user scenario. Furthermore, in a three-user scenario, BERs of $U_{2}$ and $U_{1}$ are enhanced by $2 \mathrm{~dB}$ and $9 \mathrm{~dB}$ at $10^{-6} \mathrm{BER}$, respectively. Achievable rate performance of non-cooperative and cooperative PD-NOMA are evaluated. Simulation results show that in a two-user scenario the cooperative PD-NOMA effectively enhances $U_{1}$ achievable rate by $14.1 \mathrm{bit} / \mathrm{s} / \mathrm{Hz}$ at $45 \mathrm{~dB}$ SNR. Moreover, in a three-user scenario cooperative PD-NOMA improves $U_{2}$ and $U_{1}$ achievable rates by $12.4 \mathrm{bit} / \mathrm{s} / \mathrm{Hz}$ and $14.3 \mathrm{bit} / \mathrm{s} / \mathrm{Hz}$ at $45 \mathrm{~dB}$ SNR, respectively. The recommendation for future work is to study the maximum number of users that can cooperate and achieve undisruptive communication in the proposed system. Also, we suggest extending the system for multiple LEDs and apply multiple-input multiple-output techniques to the proposed downlink cooperative PD-NOMA-VLC system and study its effect on both the BER and achievable rate performance.

Author Contributions: Conceptualization, M.A.; Validation, H.S., M.A., S.M.G. and H.E.; Formal analysis, H.S. and H.E.; Investigation, H.S.; Data curation, M.A.; Writing-original draft, H.S.; Writing-review and editing, M.A., S.M.G. and H.E.; Supervision, M.A., S.M.G. and H.E.

Funding: This research received no external funding.

Conflicts of Interest: The authors declare no conflict of interest.

\section{References}

1. Bawazir, S.S.; Sofotasios, P.C.; Muhaidat, S.; Al-Hammadi, Y.; Karagiannidis, G.K. Multiple Access for Visible Light Communications: Research Challenges and Future Trends. IEEE Access 2018, 6, 26167-26174. [CrossRef]

2. Wang, S.W.; Chen, F.; Liang, L.; He, S.; Wang, Y.; Chen, X.; Lu, W. A high-performance blue filter for a white-led-based visible light communication system. IEEE Wirel. Commun. 2015, 22, 61-67. [CrossRef]

3. Ji, R.; Wang, S.; Liu, Q.; Lu, W. High-Speed Visible Light Communications: Enabling Technologies and State of the Art. Appl. Sci. 2018, 8, 589. [CrossRef]

4. Karunatilaka, D.; Zafar, F.; Kalavally, V.; Parthiban, R. LED based indoor visible light communications: State of the art. IEEE Commun. Surv. Tutor. 2015, 17, 1649-1678. [CrossRef]

5. Mansour, A.; Mesleh, R.; Abaza, M. New challenges in wireless and free space optical communications. Opt. Lasers Eng. 2017, 89, 95-108. [CrossRef]

6. Rajbhandari, S.; Chun, H.; Faulkner, G.; Cameron, K.; Jalajakumari, A.V.N.; Henderson, R.; Tsonev, D.; Ijaz, M.; Chen, Z.; Haas, H.; et al. High-Speed Integrated Visible Light Communication System: Device Constraints and Design Considerations. IEEE J. Sel. Areas Commun. 2015, 33, 1750-1757. [CrossRef]

7. Pathak, P.H.; Feng, X.; Hu, P.; Mohapatra, P. Visible Light Communication, Networking, and Sensing: A Survey, Potential and Challenges. IEEE Commun. Surv. Tutor. 2015, 17, 2047-2077. [CrossRef]

8. Marshoud, H.; Kapinas, V.M.; Karagiannidis, G.K.; Muhaidat, S. Non-orthogonal multiple access for visible light communications. IEEE Photonics Technol. Lett. 2016, 28, 51-54. [CrossRef]

9. Wang, Z.-Y.; Yu, H.-Y.; Wang, D.-M. Energy Efficient Transceiver Design for NOMA VLC Downlinks with Finite-Alphabet Inputs. Appl. Sci. 2018, 8, 1823. [CrossRef]

10. Yin, L.; Wu, X.; Haas, H. On the performance of non-orthogonal multiple access in visible light communication. In Proceedings of the 2015 IEEE 26th Annual Symposium on Personal, Indoor, and Mobile Radio Communications (PIMRC), Hong Kong, China, 30 August-2 September 2015; pp. 1354-1359.

11. Yin, L.; Popoola, W.O.; Wu, X.; Haas, H. Performance Evaluation of Non-Orthogonal Multiple Access in Visible Light Communication. IEEE Trans. Commun. 2016, 64, 5162-5175. [CrossRef]

12. Kizilirmak, R.C.; Rowell, C.R.; Uysal, M. Non-orthogonal multiple access (NOMA) for indoor visible light communications. In Proceedings of the 4th International Workshop on Optical Wireless Communications (IWOW), Istanbul, Turkey, 7-8 September 2015; pp. 98-101. 
13. Laneman, J.N.; Tse, D.N.C.; Wornell, G.W. Cooperative diversity in wireless networks: Efficient protocols and outage behavior. IEEE Trans. Inf. Theory 2004, 50, 3062-3080. [CrossRef]

14. Taher, M.A.; Abaza, M.; Fedawy, M.; Aly, M.H. Relay Selection Schemes for FSO Communications over Turbulent Channels. Appl. Sci. 2019, 9, 1281. [CrossRef]

15. Men, J.; Ge, J. Non-Orthogonal Multiple Access for Multiple-Antenna Relaying Networks. IEEE Commun. Lett. 2015, 19, 1686-1689. [CrossRef]

16. Ding, Z.; Peng, M.; Poor, H.V. Cooperative non-orthogonal multiple access in 5G systems. IEEE Commun. Lett. 2015, 19, 1462-1465. [CrossRef]

17. Zeng, L.; O’Brien, D.C.; Minh, H.L.; Faulkner, G.E.; Lee, K.; Jung, D.; Oh, Y.; Won, E.T. High Data Rate Multiple Input Multiple Output (MIMO) Optical Wireless Communications Using White LED Lighting. IEEE J. Sel. Areas Commun. 2009, 27, 1654-1662. [CrossRef]

18. Kahn, J.M.; Barry, J.R. Wireless infrared communications. Proc. IEEE 1997, 85, 265-298. [CrossRef]

19. Komine, T.; Nakagawa, M. Fundamental analysis for visible-light communication system using LED lights. IEEE Trans. Consum. Electron. 2004, 50, 100-107. [CrossRef]

20. Wang, T.; Cano, A.; Giannakis, G.B.; Laneman, J.N. High-performance Cooperative Demodulation With Decode-and-Forward Relays. IEEE Trans. Commun. 2007, 55, 1427-1438. [CrossRef]

21. Marshoud, H.; Sofotasios, P.C.; Muhaidat, S.; Karagiannidis, G.K.; Sharif, B.S. On the Performance of Visible Light Communication Systems with Non-Orthogonal Multiple Access. IEEE Trans. Wirel. Commun. 2017, 16, 6350-6364. [CrossRef]

(C) 2019 by the authors. Licensee MDPI, Basel, Switzerland. This article is an open access article distributed under the terms and conditions of the Creative Commons Attribution (CC BY) license (http://creativecommons.org/licenses/by/4.0/). 